\title{
Spreading Code Design for a MC-CDMA Based GNSS Pilot Signal
}

\author{
Mariano Vergara $^{(1)}$, Felix Antreich ${ }^{(1)}$, Michael Meurer ${ }^{(1)}$, Gonzalo Seco-Granados
}

(1) German Aerospace Center (DLR), Germany

(2) Universitat Autónoma de Barcelona (UAB), Spain ${ }^{*}$

\begin{abstract}
Multicarrier CDMA (MC-CDMA) can represent an alternative to classical DS-CDMA for the design of GNSS ranging signals. A first requirement MC-CDMA signals must fulfill is that their generation on a satellite payload be feasible. It is well known, indeed, that multicarrier signals have a high Peakto-Average Power-Ratio (PAPR) and GNSS signals, as well as other signals used in satellite communications, must have PAPR values much lower than values usually considered acceptable in other applications. Therefore, maintaining the PAPR of a MCCDMA ranging signal within acceptable limits is a fundamental issue in any signal GNSS signal design using a multicarrier technique. The main contribution of this work is to propose a way of designing a MC-CDMA pilot signal based on Huffman codes. Such approach allows to shape the autocorrelation (or equivalently the power spectrum) of the pilot signal according to the specific needs of a GNSS service, guaranteeing arbitrary small PAPR values in all cases.
\end{abstract}

\section{INTRODUCTION}

Multicarrier (MC) transmission is a transmission technique that is developing very fast and getting the upper hand in the design of many communication systems and may be a candidate also for new GNSS signal designs. In particular, the MC-CDMA transmission scheme represents a spread spectrum technique characterised by a spreading operation in the frequency rather than time domain. For this reason, it can be viewed as a dual technique to DS-CDMA. A previous study [1] has proven that a pilot signal based on this technique can achieve a similar synchronization accuracy than a single carrier DS-CDMA ranging signal. Nevertheless, a major obstacle on the path for this technology to be taken into consideration for future GNSS signal designs is the high Peak-to-Average Power Ratio (PAPR), that is inherent to all multicarrier modulations. Achieving very low PAPR values is of paramount importance for GNSS applications, as well as for any other satellite communication, where the available transmit power is limited and the Signal-to-Noise Ratio (SNR) very low. A signal with a high PAPR is very likely to undergo nonlinear distortions. This entails spectral growth of the multicarrier

* The work of prof. G. Seco-Granados has been partially supported by the Spanish Government under project TEC2008-06305, and by the Chair of Knowledge and Technology Transfer Parc de Recerca UAB - Santander. G. Seco-Granados is also affiliated with IEEC-UAB. signal in the form of intermodulation products, which are the cause of out-of-band radiations and interference with other systems. In order to avoid this pernicious effect, one would be forced to reduce the average radiated power, with obvious consequences on the SNR and the quality of the service. A way to overcome this deadlock consists in lowering the PAPR of the MC signal with a careful choice of the symbols to be transmitted. In a MC-CDMA pilot, the trasmitted symbols are the chips of the spreading sequence, and not information symbols, as in multicarrier transmission systems. In this latter case, the almost totality of PAPR minimization techniques consist in mapping the information symbols to other symbols that can be transmitted with a lower PAPR [2]. This mapping has to be performed in real-time within the symbol interval and is limited by the maximum number of IFFT operations possible to perform in the unit time. In MC-CDMA signal design, the choice of the optimal spreading sequences to be transmitted can be carefully made offline, with neither time nor computation constraints. The PAPR minimization problem in MC-CDMA can thus be transformed into the problem of finding a suitable family of spreading codes capable of bringing the PAPR down to values which are comparable to the PAPR values of the current GALILEO signals. The spreading sequence, on the other hand, also affects the spectrum and the autocorrelation of the MC-CDMA signal and the choice of the spreading sequence must be done also according other criteria than PAPR alone. There are several properties that are to be wished from a GNSS ranging signal; tracking accuracy (low tracking jitter) is one of them, but not always the most relevant. In some other cases the robustness of the tracking may be more important, in some others interference resilience or short acquisition times can be more preferable. Besides, interoperability and compatibility with an increasing number of GNSS systems sharing the same frequency bands is gaining a significant role in the near future. Multicarrier transmission technique enables the optimization of one or some of the aforementioned properties by simply changing the power allocation amongst the subcarriers. The here presented signal design based on Huffman codes enables to shape the power spectrum and the autocorrelation of the ranging signal, guaranteeing an arbitrary small PAPR in all cases. The alluring aspect of all this is that with such a MC-CDMA signal design a great amount of flexibility is added to GNSS signal design, 
insomuch as modifying the ranging signal properties would not alter the receiver or payload design, but it would only entail a redistribution of transmitted power amongst the subcarriers.

\section{Signal Model}

Amongst the possible combinations of Multicarrier modulation and CDMA [3], we have chosen the classical MC-CDMA variant: the users share the same frequency band and are separated by spreading codes in frequency domain. We assume that the length of the code and the number of subcarriers are equal. Let our transmitted signal be:

$$
x(t)=\sqrt{P} \sum_{k=-\infty}^{\infty} \sum_{m=1}^{M} c_{m}^{u} g(t-k T) e^{j 2 \pi m \Delta t}
$$

where :

$M \quad$ : number of subcarriers.

$P \quad:$ transmitted power.

$\mathbf{c}^{u} \quad$ : spreading code assigned to the user u. $\mathbf{c}^{u}=$ $\left[c_{1}^{u}, \ldots, c_{M}^{u}\right]$.

$\Delta \quad$ : frequency separation amongst the subcarriers (equal for all of them).

$g(t) \quad$ : impulse response of the transmitting filters (same for all filters).

We assume that the pulse $g(t)$ has unitary energy:

$$
\int_{\infty}^{\infty}|g(t)|^{2} \mathrm{~d} t=\int_{-B}^{B}|G(f)|^{2} \mathrm{~d} f=T
$$

with $G(f)$ being the Fourier Transform of $g(t)$ and B its bandwidth. The condition that B must fulfill is:

$$
B \leq \frac{\Delta}{2}
$$

Further, it must hold:

$$
\left\|\mathbf{c}^{u}\right\|_{2}^{2}=\sum_{m=1}^{M}\left|c_{m}^{u}\right|^{2}=1
$$

The orthogonality amongst the M subchannel can be obtained by any appropriate choice of prototype transmit filters and frequency separation. The only requirement of this model is that the all transmitting filters have the same phase characteristic.

We define the PAPR of a non-zero signal $x(t)$ as:

$$
P A P R=\frac{\|x(t)\|_{\infty}^{2}}{\mathbb{E}\left[|x(t)|^{2}\right]}
$$

with:

$$
\|x(t)\|_{\infty}^{2}=\max _{t}\left\{|x(t)|^{2}\right\}
$$

As long as condition :

$$
\int_{-\infty}^{\infty}\left|g(t)\left[\sum_{m=1}^{M} c_{m} e^{j 2 \pi m \Delta t}\right]\right|^{2} \mathrm{~d} t=\Psi \quad, \forall \mathbf{c}_{m}
$$

holds, the average power of the signal $x(t)$ does not depend the spreading sequence. Minimising the PAPR is thus equivalent to minimising the peak value. Interpreting the results of [4], we can write the latter as:

$$
\|x(t)\|_{\infty}^{2}=P \Gamma_{g} \max \left|\sum_{m=1}^{M} c_{m}^{u} e^{j 2 \pi m \Delta t}\right|^{2}
$$

where $\Gamma_{g}$ is a factor that depends on the pulse shape $g(t)$. The term $\left|\sum_{m=1}^{M} c_{m}^{u} e^{j 2 \pi m \Delta t}\right|^{2}$ is the $\infty$-norm of $x(t)$ when the pulse $g(t)$ is chosen to be a rectangular window for all subcarriers. When all the pulses are chosen equal, the rectangular pulse is the one that brings about the lowest peak value; for all the other cases it can be proven that $\Gamma_{g}>1$ [4]. All PAPR values discussed in this work are for $\Gamma_{g}=1$.

It is our interest to find a spreading code family that minimises the PAPR and at the same time makes the signal $x(t)$ suited for ranging in a GNSS scenario. Differently from DS-CDMA, MC-CDMA signals have an autocorrelation function that is strongly dependent on the spreading code, rather than on the pulse shape. With some mathematical passages, we obtain the autocorrelation of the signal $x(t)$ :

$$
\begin{aligned}
R_{x}(\tau) & =\frac{1}{T} \int_{T} x(t) x^{*}(t+\tau) \mathrm{d} t \\
& =\frac{P}{T} \mathfrak{F}\left\{\left|c_{m}^{u}\right|^{2}\right\} \sum_{k=-\infty}^{\infty} R_{g}(\tau+k T)
\end{aligned}
$$

with $\mathfrak{F}\{\cdot\}$ denoting the Discrete-Time Fourier Transform (DTFT), where the variable in the transformed domain is replaced by $\Delta \tau ; R_{g}(\tau)$ is the autocorrelation function of the shaping pulse $g(t)$. If the bandwidth B is such that, besides (3), also this condition holds:

$$
B \leq \frac{1}{T}
$$

then:

$$
\sum_{k=-\infty}^{\infty} R_{g}(\tau+k T)=\frac{1}{T}|G(0)|^{2}
$$

and it is possible to say that, practically, the pulse shape does not play any role into the autorrelation function of the ranging signal. In the following representation we assume this constant to be unitary. Condition (10) applies for a root-raised cosine pulse of arbitrary roll-off factor. Eq. (9) is a generalization of the expression to be found in [5]. 
Since we have adopted a baseband equivalent signal model, the autocorrelation (9) is a complex quantity. We note also that all the PAPR values mentioned in this work are calculated for a baseband equivalent signal. A modulated signal always has a higher PAPR; while a sinusoid has a PAPR of $3 \mathrm{~dB}$, for example, a complex exponential has a PAPR of $0 \mathrm{~dB}$. The relationship between the PAPR of a baseband and a passband signal is neither investigated nor discussed here ${ }^{1}$.

\section{ZADOFF-CHU CODES}

If we are looking for spreading codes for MC-CDMA with very low PAPR, then polyphase Zadoff-Chu codes are the first choice [6]. In particular, if we superimpose the additional condition that the power is distributed uniformly amongst the subcarriers :

$$
\left|c_{k}^{u}\right|^{2}=\frac{1}{M} \quad \forall k \quad,
$$

Zadoff-Chu represent certainly the best solution up to the current date. Indeed, minimising PAPR with the side condition (12) brings us to a very old mathematical problem that is still open to our knowledge [8]-[9]: how to choose the phases of a multitone signals so that its peak value is minimum. The best solution to this problem, although only empirical, is currently provided by the so called chirp-like sequences [10]-[11]: sequences whose phases vary quadratically with the code element index. Chu [12] and Zadoff [13] proposed a way to generate a whole family of codes with this property ${ }^{2}$. A Zadoff-Chu code [12] of length $M$ is built as:

$$
c_{k}=\left\{\begin{array}{ll}
e^{j \frac{2 \pi r k^{2}}{N}} & \mathrm{~N} \text { even } \\
e^{j \frac{2 \pi r k(k+1)}{N}} & \mathrm{~N} \text { odd }
\end{array} \quad k=0,1, \ldots, N-1\right.
$$

where $\mathrm{r}$ is an integer relatively prime to $\mathrm{N}$.

A simple way to generate a Zadoff-Chu code family is to choose $N$ a prime number and then let $r$ vary from 1 till $N-1$. Each code obtained for a specific $r$ is assigned to a user. As shown in Fig. 1 for $N=31$, each Zadoff-Chu code has a PAPR which depends on the code index $r$. The PAPR for $r=1$ corresponds to the PAPR of the Newman sequences, that is always approximately $2.6 \mathrm{~dB}$ independently from the code length [9]. Having codes with different PAPRs can be a disadvantage if we want to assign each code to a satellite. However, in spite of having different PAPRs, MC-CDMA pilot

\footnotetext{
${ }^{1}$ In [7] a factor 2 (equivalent to $3 \mathrm{~dB}$ ) is mentioned, but this seems to hold only in the case of multicarrier transmission using QAM modulation.

${ }^{2}$ However it must be mentioned that Zadoff, Chu and Frank have worked at another problem: how to generate a code with ideal cyclic autocorrelation. That choosing the phases of a multitone signal according chirp-like sequences yields very low PAPRs was noticed only later, and to the authors knowledge, for the first time in [8].
}

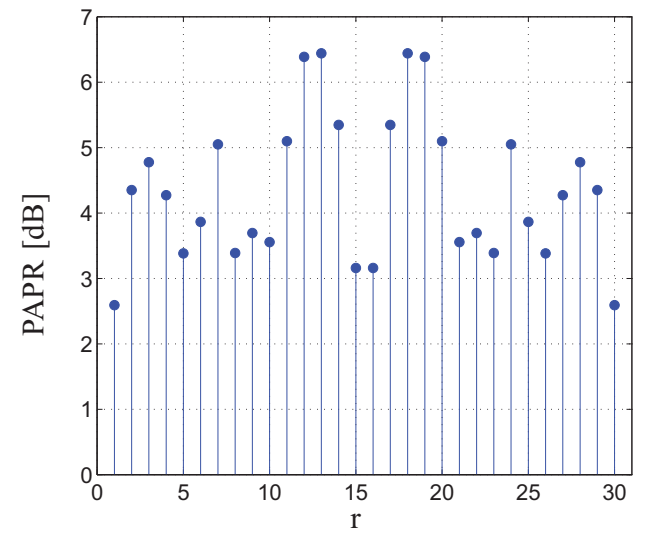

Fig. 1. PAPR of MC-CDMA signals using a Zadoff-Chu code family of length $\mathrm{N}=31$.

signals based on a family of Zadoff-Chu codes have the same autocorrelation function, because of (9) and (12). In Fig.2 we have plotted the autocorrelation of the signal (1) when $\mathbf{c}_{m}^{u}$ is chosen to be a Zadoff-Chu code of length $\mathrm{N}=31$. If for any reason the spectrum of the ranging signal is required to be flat, Zadoff-Chu codes represent the best choice that can be made to minimise the PAPR. A limitation of the Zadoff-Chu codes is that if we want more codes with low PAPR we have to increase the number of subcarriers. A even greater limit is that condition (12) prevents us from shaping the signal spectrum as we like. One of the advantages of Zadoff-Chu codes is that they provide at the same time also a relatively low average bit-error probability [6], [15]. While this is not important for a pilot signal, it might turn helpful for GNSS signals used for data transmission, rather than for synchronization.
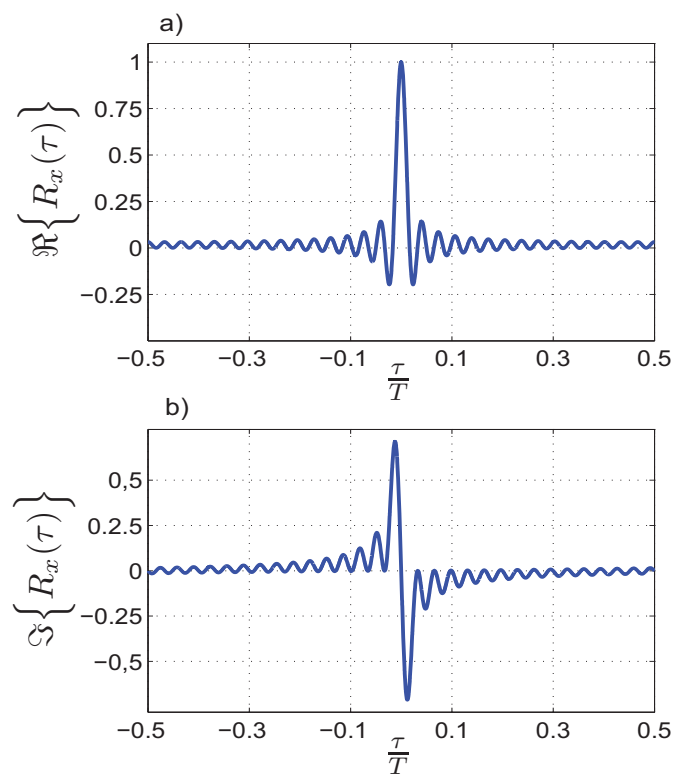

Fig. 2. Real (a) and imaginary (b) part of the autocorrelation of a MC-CDMA pilot signal based on Zadoff-Chu Codes of length $\mathrm{N}=31$. 


\section{HuffMAn CODES}

Unlike a DS-CDMA system, a MC-CDMA system can employ non-constant envelope (or multilevel) spreading sequences without extending the dynamic range and the PAPR, as noted in [6]. This observation opens new horizons for the search of candidate spreading sequences for a MC-CDMA based GNSS signal. Huffman codes are multilevel sequences and were developed for for pulse compression in radar systems [16], [17]. Their possible use as spreading sequences for MCCDMA was first suggested by Popović in [6] and [14]. A Huffman code of length $N=M+1$ is a finite sequence of complex numbers $\mathbf{c}=\left\{c_{M}, c_{M-1}, \ldots, c_{0}\right\}$ with the following aperiodic autocorrelation function:

$R_{\text {code }}(d)=\sum_{k}^{M-d} c_{k} c_{k+d}^{*}\left\{\begin{array}{l}=1 \quad, \text { for } d=0 \\ =0 \quad, \text { for } d=1,2, \ldots, M-1 \\ =\frac{1}{\gamma} \neq 0 \quad, \text { for } d=M\end{array}\right.$

We indicate the code autocorrelation by $R_{\text {code }}$, to avoid confusion with the autocorrelation of the MC-CDMA signal in (9). Without loss of generality we can consider codes whose energy is normalised to one. The value of the autocorrelation for shifts $d= \pm M$ is called edge side-lobe and it is a code parameter. In our notation, $\gamma$ represents the central-to-side lobe ratio of the code autocorrelation. Huffman codes are obtained by means of polynomial representation. Let:

$$
P(s)=c_{M} s^{M}+c_{M-1} s^{M-1}+\ldots+c_{1} s+c_{0}
$$

be the polynomial representing the code $\mathbf{c}=$ $\left\{c_{M}, c_{M-1}, \ldots, c_{0}\right\}$. A polynomial of degree $M$, represents a code of length $N=M+1$. In order for such a code to have a autocorrelation equal to the one in (14), the roots of the corresponding polynomial must fulfill two conditions :

1) The roots $\left\{z_{n}\right\}$ must be equally spaces in the complex plane and the angular spacing must be $\frac{2 \pi}{M}$.

2) For each angle a root must be on (only) one of two circles, whose radii are (see also [17]):

$$
R_{1,2}=\left|c_{0} c_{M}^{*}\right|^{-1}\left[\left|\frac{\gamma}{2}\right| \mp \sqrt{\frac{\gamma^{2}}{4}-1}\right]^{\frac{1}{M}}
$$

In some works [18]-[20] the complexity of the code generation is limited by making several simplifying assumptions. On the other hand, together with the complexity, also the cardinality of the code family is limited. We believe that by doing so, some codes of interest to us can be left out. If for example we generate only real Huffman codes, we consider only the codes with the worst possible configuration of phases: same phase for all subcarriers is the choice of phases that maximises the PAPR. Hence, we made only the following assumptions. The first one does not entail any loss of generalization:

$$
c_{M}=1
$$

the only limiting assumption we made is:

$$
c_{0}=\mp c_{M}
$$

This assumption makes it possible to interpret the two radii in (16) as one being the reciprocal of the other and it cancels the dependency of the radii on the coefficients that are the unknown variables of our problem. The number of Huffman codes of a given length $\mathrm{N}$ that can be generated in this way is:

$$
\Omega=\left(\begin{array}{c}
N-1 \\
\frac{N-1}{2}
\end{array}\right)
$$

The two parameters $N$ and $\gamma$ define a Huffman code family of $\Omega$ codes. We indicate a Huffman code family with the notation: $\mathcal{H}(N, \gamma)$. Codes belonging to the same family have the same (code) aperiodic autocorrelation. It is possible to prove that spreading sequences with the same aperiodic code autocorrelation produce MC-CDMA signals with the same PAPR. The relationship that connects the PAPR of a signal (1) based on a Huffman code of the family $\mathcal{H}(N, \gamma)$ and the parameter $\gamma$ is ${ }^{3}$ :

$$
\operatorname{PAPR}_{H u f f}(\gamma)=10 \log _{10}\left(1+\frac{2}{\gamma}\right) \quad \mathrm{dB}
$$

This implies that with the Huffman codes is possible to generate MC-CDMA codes with an arbitrary small PAPR. As the value of $\gamma$ approaches infinity, the PAPR tends to $0 \mathrm{~dB}$, which is the resulting PAPR when all the power is allocated to only one subcarrier. Thus, the trade off is as follow: the lower the PAPR, the more unbalanced the power distribution amongst the subcarriers. Such a degree of flexibility is not given by any other code. An interesting property of the Huffman codes is that within a family $\mathcal{H}(N, \gamma)$ there are several codes that have an identical distribution of energy amongst the code elements and they only differ in the phases. In terms of MC-CDMA signals, this means that it is possible to generate several signals with the same power profile (i.e. power distribution amongst the subcarriers), that is to say, signals with the same power spectrum. Huffman codes with the same energy distribution form a subset within the overall family of $\Omega$ codes. To have a better understanding of that, we present an example. Let us consider the Huffman family $\mathcal{H}(N=11, \gamma=11)$. Out of the 252 codes, let us pick three codes with different energy distribution. For each code we create a MC-CDMA signal of the type (1). For each energy distribution there exist also

\footnotetext{
${ }^{3}$ This can be obtained following [6].
} 
other codes, that differ amongst them only in the phases of the code elements. In Fig.3a. the energy distribution of three sample codes. Equivalently this represents the power profile of the corresponding MC-CDMA signals. In Fig. 3b we depict the real part of the autocorrelations of the MC-CDMA signals around zero is depicted. The delay $\tau$ is normalised to the inverse of the MC symbol rate. By $R_{n}$ we indicate the autocorrelation of the MC-CDMA signal that uses the code $c_{n}$, for $n=1,2,3$. The signal based on the code $c_{1}$ has more power on the subcarriers at the edge of the spectrum, and as such, the corresponding autocorrelation is the steepest one of the three; the signal based on the code $c_{2}$ does just the opposite and concentrates most of the power onto the subcarriers at the center of the spectrum. If the only property we want from our ranging signal is low tracking jitter, then the code $c_{1}$ is the best one amongst the three. But if in some GNSS service tracking robustness is preferred over tracking robustness, then the code $c_{2}$ is the best one amongst the three. As it can be seen in Fig. 4, the signal built on the code $c_{2}$ is the one with the autocorrelation function with the smallest number and levels of side-peaks. For services that require both tracking accuracy and tracking robustness, the code $c_{3}$ can be the optimal choice. As long as we can formulate the desired properties in terms code elements, we can look for the code subset (same energy distribution) which comes closest to our needs, and allocate each code of the subset to a different satellite. In case this were not sufficient, because the number of code subsets is limited, it is possible to change the parameter $\gamma$. This will change the energy distribution of all subsets of codes and with it the power spectrum of the obtainable MC-CDMA. In such a way, we can bargain the PAPR with the desired ranging signal properties.

\section{CONCLUSION}

The main aim of the work was to introduce another optimization criterion beside tracking accuracy in the design of Multicarrier-based GNSS ranging signals : low PAPR. This can be minimised by a careful choice of the spreading sequence. Unlike the case of traditional DS-CDMA, the spreading sequence of a MC-CDMA signal affects also its power spectrum and its autocorrelation. A first design scheme based on Zadoff-Chu codes delivers low PAPR, but reduced flexibility. On the other hand the design based on Huffman codes seems extremely promising, because it allows to to select the spreading sequence that most suits the application, out of a set of sequences that all have the property of producing an arbitrarily small PAPR. Huffman sequences can be seen as a catalogue of possibilities we have for setting the powers and the phases of the subcarriers, in order to achieve a certain PAPR, which depends exclusively the code parameter $\gamma$ by means of a very simple relation. In a Huffman code family there are several codes that share the same distribution of energy amongst the code elements, and as such, generate MCCDMA signals with equal power spectrum and autocorrelation
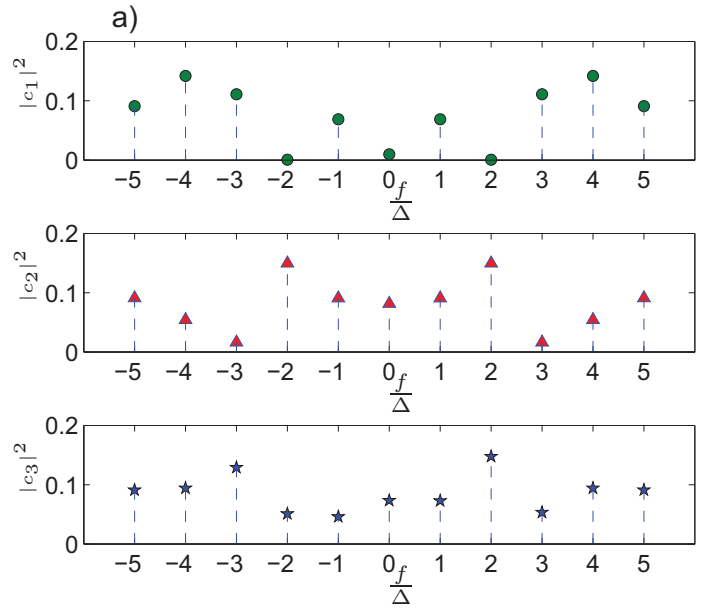

b)

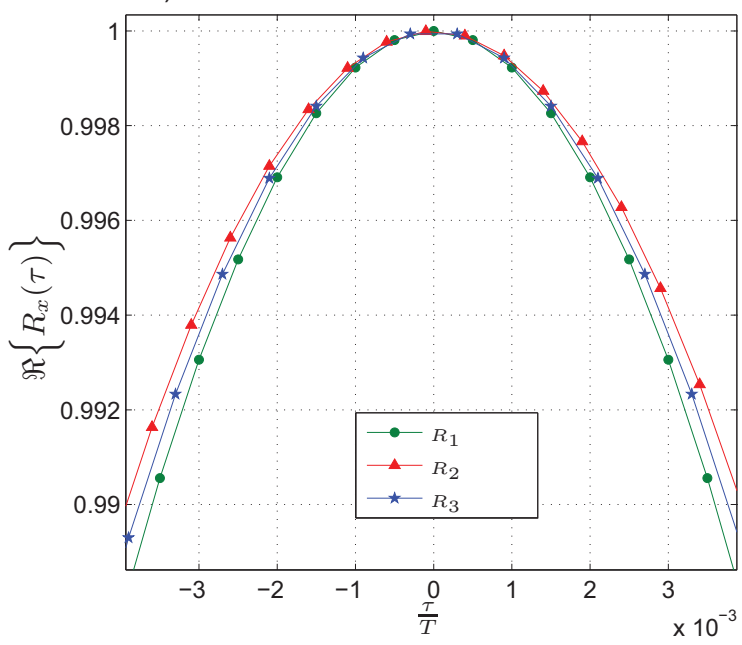

Fig. 3. (a) Power profile of 3 MC-CDMA signals obtained with 3 different Huffman codes $\mathcal{H}(11,11)$, and (b) their corresponding autocorrelation function (real part) around the zero. All three signals have a PAPR of 0.7255 dB.

function. As a consequence of all that, once can envisage to assign to each GNSS service a subset of the Huffman family $\mathcal{H}(N, \gamma)$ with the same energy distribution, and assign to every a satellite a code of this subset. All this procedure is extremely flexible and can be easily changed over time, since it depends only on the power and the phases of the individual subcarriers.

\section{ACKNOWLEDGMENT}

The authors would like to thank Marc Cassolá Rodríguez, Matteo Sgammini and Nikola Basta from DLR. The discussions with them were of significant value in the development of this work. Moreover, we thank prof. Mario Tanda from the technical university of Naples for a helpful review.

\section{REFERENCES}

[1] F. Zanier, M. Luise, "Fundamental Issues in Time-Delay Estimation of Multicarrier Signals with Applications to Next-Generation GNSS", 10th 
a)

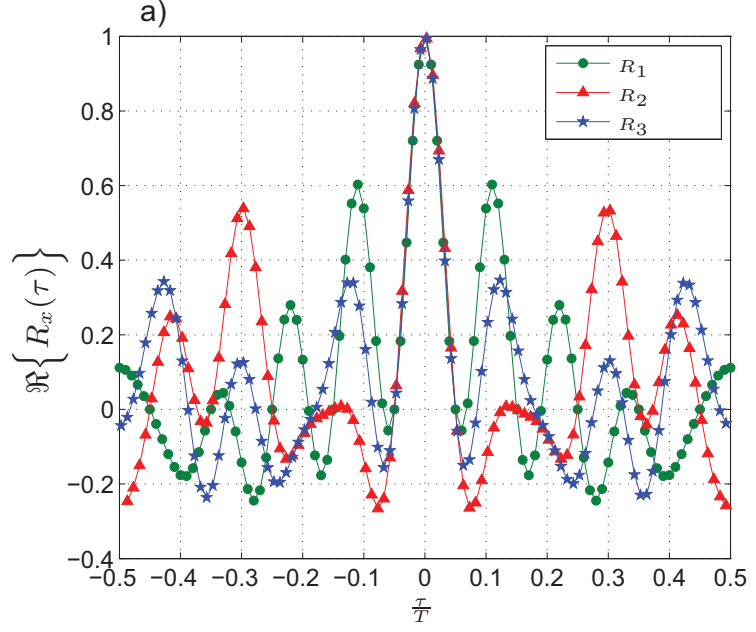

b)

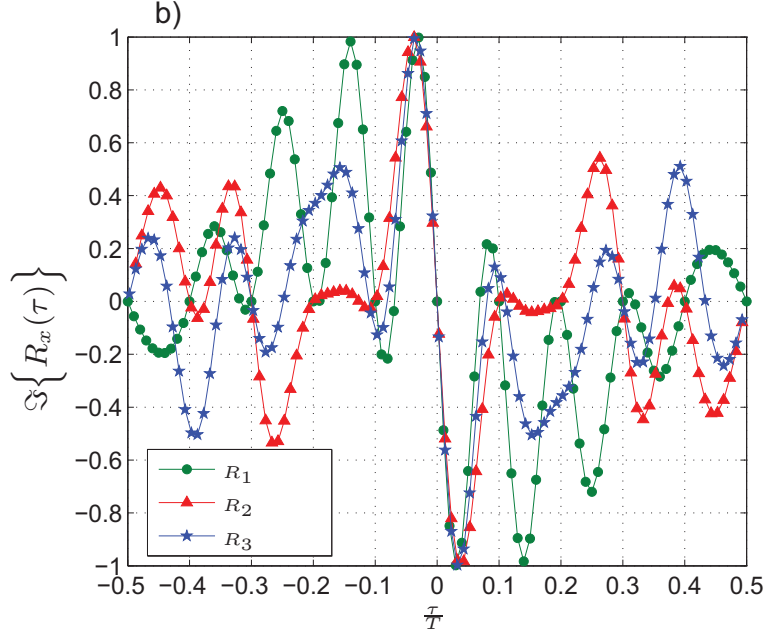

Fig. 4. Real (a) and imaginary (b) part of the autocorrelation functions of the three codes in Fig. 3a

International Workshop on Signal Processing for Space Communications, SPSC 2008.

[2] S.H. Han and J. H. Lee, "An Overview of Peak-to-average Power Ratio Reduction Techniques for Multicarrier Transmission", IEEE Wireless Comm. Mag., pp. 56-65, Apr. 2005.

[3] L. Yang, L. Hanzo, "Multicarrier DS-CDMA:A Multiple Access Scheme for Ubiquitous Broadband Wireless Communications", IEEE Communication magazine, pages 116-124, October 2003.

[4] S. Ben Slimane, "Peak-to-average power ratio reduction of OFDM signals using pulse shaping", VTC '98, USA, 1998.

[5] J. H. Yooh and V. K. Wei, "On synchronizing and detecting multi-carrier CDMA signals", in Proc. ICUPC 95, Tokyo, Nov. 1995, pp. 512-516.

[6] B. M. Popovic, "Spreading Sequences for Multicarrier CDMA Systems", IEEE Trans. on communications, Vol. 47, No. 6, June 1999.

[7] J. Tellado, Multicarrier Modulation with Low PAPR, Kluwer Academic Publishers, 2000.

[8] S. Boyd, "Multitone signals with low crest factor", IEEE Trans. Circuits Syst., vol. CAS-33, pp. 1018-1022, Oct. 1986

[9] D. R. Gimlin, C. R. Patisaul, "On Minimizing the Peak-to-Average Power Ratio for the Sum of N Sinusoids", IEEE Trans. on Communiations, Vol. 41, N0. 4, April 1993.

[10] D.I. Newman, "An L1 extrema1 problem for polynomials", Proc. Amer. Math. Society, vol 16 pp 1287-1290, Dec. 1965.

[11] M. R. Schroeder, "Synthesis of Low-Peak-Factor Signals and Binary
Sequences With Low Autocorrelation", IEEE Trans. Inform. Theory (Corresp.), vol. IT-16, pp. 85-89, Jan. 1970.

[12] D. C. Chu, "Polyphase codes with good periodic correlation properties", IEEE Trans. Inform. Theory, vol. IT-18, pp. 531-532, July 1972. 1972.

[13] R. L. Frank and S. A. Zadoff, "Phase shift pulse codes with good periodic correlation properties ", IRE Trans. Inform. Theory (Corresp.), vol. IT-S, pp: 381-382, Oct. 1962

[14] B. M. Popovic, "Spreading Waveforms for Multicarrier CDMA Systems", Proc. of Spread Spectrum Techniques and Applications, Vol. 1, pages 205-209, Sept. 1998.

[15] P. Drotár, J. Gazda, P. Galajda and D. Kocur, "Joint Microstatistic Multiuser Detection and Cancellation of Nonlinear Distortion Effects for the Uplink of MC-CDMA Systems Using Golay Codes", International Journal of Electronics, Communications and Computer Engineering, vol. 1, no. 2, pp. 87-93, Paris, France, 2009.

[16] David A. Huffman, "The generation of impulse equivalent pulse trains", IRE. Trans. Inform. Theory, vol. ITS, no. 5, pp. 10-16, 1962.

[17] Madav Levanon and Eli Mozeson, Radar signals, John Wiley \& Sons, Inc., 2004.

[18] M. H. Ackroyd, "Huffman sequences with approximately uniform envelopes or cross-correlation functions", IEEE Trans. Inform. Theory, vol. IT-23, no. 5, pp. 620-623, Sept. 1977.

[19] P.S. Mohahir , "Multilevel aperiodic Huffman sequences2, Electronics Letters, Vol. 11 No.3, February 61975.

[20] J. M. Hunt and M. H. Ackroyd, "Some integer Huffman sequences", IEEE Trans. Information Theory, vol. IT-26, pp. 105-107, Jan. 1980 